\title{
Betrayal Trauma and Borderline Personality Characteristics: Gender Differences
}

\author{
Laura A. Kaehler and Jennifer J. Freyd \\ University of Oregon
}

\begin{abstract}
Borderline Personality Disorder has been linked to both trauma and insecure attachment styles. Betrayal Trauma Theory proposes those who have experienced interpersonal trauma may remain unaware of betrayal in order to maintain a necessary attachment. This study attempts to replicate the association between self-reported betrayal trauma experiences and borderline personality characteristics found by Kaehler and Freyd (2009); however, this project includes participants from a community, rather than a college, sample. Using multiple regression, all three levels of betrayal (high, medium, and low) and gender were significant predictors of borderline personality characteristics. Separate regression analyses were conducted for both genders to explore the associations of betrayal trauma on these traits. For men, all three levels of betrayal trauma were significant predictors; for women, only high and medium betrayal traumas were significant. These findings suggest trauma may be a key factor of borderline personality disorder, with differential effects for betrayal and gender.
\end{abstract}

Keywords: borderline personality disorder, betrayal trauma, gender

The defining characteristics of Borderline Personality Disorder (BPD) are volatile interpersonal relationships, identity confusion, pronounced emotional lability, and poor impulse control. Prevalence rates for this serious mental disorder are approximately $2 \%$ of the general population, $10 \%$ of psychiatric outpatients, and 15-20\% of clinical inpatients (Diagnostic and statistical manual of mental disorders, 4th ed-text revision; APA, 2000). Yet, an interesting gender effect has been observed with this disorder: $75 \%$ of those diagnosed are women $(D S M-I V-T R)$ and approximately $80 \%$ of clients receiving treatment for BPD are women (Skodol et al., 2002). Due to both the large numbers of people affected by this disorder and the sociocultural factors associated with BPD, many theories have been suggested regarding the causal factors of this disorder, including the roles of trauma and attachment.

One consistent finding regarding BPD and attachment is the association between BPD and insecure attachment styles (Levy, 2005). In fact, some have suggested a disorganization of the attachment system is a key contributor of BPD features (Gunderson \& Lyons-Ruth, 2008). A review of studies exploring this association by Agrawal, Gunderson, Holmes, and Lyons-Ruth (2004) revealed that adults with BPD most frequently display either fearful or unresolved (with a secondary classification of preoccupied) attachment styles. A fearful attachment style is characterized by a desire for intimacy, while simultaneously fearing hurt or rejection by the partner. Like the fearful attachment style, a person with an unresolved/preoccupied attachment style also seeks an intimate relationship, but is instead sensitive to a per-

This article was published Online First August 15, 2011.

Laura A. Kaehler and Jennifer J. Freyd, Department of Psychology, University of Oregon.

Correspondence concerning this article should be addressed to Laura A. Kaehler and Jennifer J. Freyd, Department of Psychology, University of Oregon, Eugene, OR 97403-1227. E-mail: jjf@uoregon.edu ceived dependency on the partner. A link between infant insecure attachment and subsequent development of BPD symptoms has also been demonstrated (Lyons-Ruth, Yellin, Melnick, \& Atwood, 2005; Rogosch \& Cicchetti, 2005). Recent findings from a prospective longitudinal study (Carlson, Egeland, \& Sroufe, 2009) showed that a disorganized infant attachment style, which includes a sequential or simultaneous display of contradictory approach/ avoid behaviors, predicted adult borderline symptoms. A hallmark feature of relationships with persons diagnosed with BPD is inconsistencies in thoughts and actions (e.g., "push-pull dynamics"), similar to the descriptions of attachment styles mentioned above.

Parental abuse and neglect interferes with the development of a secure attachment style (Baer \& Martinez, 2006; Lamb, Gaensbauer, Malkin, \& Schultz, 1985). For example, Minzenberg, Poole, and Vinogradov (2006) found all types of childhood maltreatment to be significantly associated with attachment-avoidance, with childhood sexual abuse (CSA) also related to attachment-anxiety. These are classic indicators of insecure attachment. Rates of maltreatment as high as approximately $90 \%$ have been found in BPD patients (Zanarini et al., 1997). Looking at CSA specifically, the prevalence rate of this trauma has been estimated to be as high as $75 \%$ in individuals with BPD, including both inpatient and outpatient samples (Battle et al., 2004). It should be noted that children who have experienced CSA are also at increased risk for experiencing other forms of childhood interpersonal violence, for example, domestic violence (Bowen, 2000) and physical abuse (Zanarini, 2000). In fact, physical abuse, emotional abuse, and neglect are frequently observed and are all associated with BPD (Battle et al., 2004; Carlson et al., 2009; Herman, Perry, \& van der Kolk, 1989; Johnson, Smailes, Cohen, Brown, \& Bernstein, 2000; Paris, 1997; Trull, 2001). Given the demonstrated links among trauma, insecure attachment, and BPD, a parsimonious model in which to explore BPD would incorporate both attachment and trauma. Betrayal Trauma Theory (BTT; Freyd, 1996) is a concep- 
tual framework that posits attachment as central to the impact of trauma.

Bowlby (1988, p. 121) suggests the attachment relationship "has a key survival function of its own, namely protection." BTT utilizes that premise to propose individuals may isolate knowledge about betrayals, such as those that occur during maltreatment, in order to maintain a relationship necessary for survival (Freyd, 1996). Typically, it would be advantageous to be able to detect betrayal in order to prevent a future violation; however, one may find it more adaptive to remain unaware of that violation if that knowledge threatens a person's more immediate viability (Freyd, 1996). Specifically focusing on the parent-child relationship for a moment, the parent is solely responsible for ensuring the physical and psychological needs of the child are met. Thus, if the parent betrays the child - for example, via physical abuse - the child must still remain attached to the caregiver (and ensure the caregiver is attached to the child) in order to survive. Although a natural response to betrayal is to withdraw, if the child would react in that manner, his or her life would be in peril. Therefore, the child may remain unaware of that betrayal in an effort to maintain that necessary connection to the parent. Freyd (1996) has suggested one mechanism by which this knowledge isolation may occur is dissociation, defined by Bernstein and Putnam (1986, p. 727) as "a lack of normal integration of thoughts, feelings, and experiences into the stream of consciousness and memory." Interestingly, severe dissociation is one of the nine diagnostic criteria for BPD and some have suggested it is a key, distinguishing component of BPD (Ross, 2007; Skodol et al., 2002; Wildgoose, Waller, Clarke, \& Reid, 2000; Zweig-Frank, Paris, \& Guzder, 1994).

Kaehler and Freyd (2009) explored the association between borderline personality characteristics and betrayal trauma experiences within a college population. Using the Brief Betrayal Trauma Survey (BBTS; Goldberg \& Freyd, 2006) and a modified version of the Borderline Personality Inventory (BPI; Leichsenring, 1999), results showed high- and medium-betrayal traumas significantly predicted borderline personality characteristics, while low-betrayal traumas did not. Interestingly, these results were found while controlling for gender, which was not associated with borderline personality characteristics.

The authors suggest the gender differences observed in BPD diagnoses may be attributable to the nature of the trauma experienced, rather than differences between genders. As reported in Goldberg and Freyd (2006), women tend to experience more high-betrayal traumas, while men experience more low-betrayal events. Work by Johnson and colleagues (2003), as part of the Collaborative Longitudinal Personality Disorders Study (CLPS), support this interpretation. These researchers compared men and women who met diagnostic criteria for BPD and found no significant differences between the two groups for rates of childhood sexual abuse, physical abuse, and witnessing abuse. However, the authors do not report on the closeness of the relationship to the perpetrator, so it is not possible to differentiate between the levels of betrayal present. A recent study by Woodward, Taft, Gordon, and Meis (2009) showed that clinicians, when evaluating ambiguous case vignettes of a person who experienced childhood sexual abuse that included symptoms of both BPD and PTSD, were not more likely to assign women the BPD diagnosis. Thus, clinicians were responding to the nature of the trauma, not the client's gender, when interpreting the case.
In the current study, we attempt to replicate the findings of Kaehler and Freyd (2009) using a community sample. Thus, it was predicted that high- and medium-betrayal trauma would be associated with borderline personality characteristics (with highbetrayal as the stronger predictor), while low-betrayal trauma would not be a significant predictor.

\section{Method}

\section{Participants}

Participants were members of the Eugene-Springfield Community Sample (ESCS), who were recruited via direct mailings from a list of homeowners in 1993. Since then, this group has been sent questionnaires yearly; in 2000, the 16-page questionnaire included items related to trauma history and borderline personality characteristics. Of the approximately 850 participants who were administered this questionnaire, 749 participants (57\% women) completed them. The mean age of the sample was 50.7 years old $(S D=$ 12.6). The sample predominantly identified as Caucasian $(96.0 \%)$ and a majority was married $(80 \%)$. This was a highly educated (23\% postgraduate degrees) sample, with $43 \%$ of participants employed full-time (20\% retired). Please see Goldberg (1999a, 1999b) and Goldberg and Freyd (2006) for additional information regarding the ESCS.

\section{Materials and Procedure}

As in the Kaehler and Freyd (2009) study, the Brief Betrayal Trauma Survey (BBTS; Goldberg \& Freyd, 2006) and a modified version of the Borderline Personality Inventory (BPI; Leichsenring, 1999) were used. The BBTS is a 12-item, self-report measure of major traumatic events participants may have experienced during two time periods (before and after age 18). Each item is classified as having one of three levels of betrayal: low, medium, or high. Noninterpersonal traumas (e.g., motor vehicle accidents) are viewed as a low-betrayal, while interpersonal traumas (e.g., being attacked) are considered a medium- or high-betrayal. To distinguish high-betrayal items from medium-betrayal items, the closeness of the perpetrator is assessed. An example of a lowbetrayal item is "been in a major motor vehicle accident that resulted in significant loss of personal property, serious injury, death, or fear of own death." An example of a medium-betrayal item is "you were deliberately attacked so severely as to result in marks, bruises, blood, or broken bones by someone with whom you were not close [italics added]." An example of a high-betrayal item is "you were deliberately attacked that severely by someone with whom you were very close [italics added]." Respondents select the frequency of the event from three options ("never", "one or two times", or "more than that"). The BBTS has been demonstrated to have both good construct validity (DePrince \& Freyd, 2001) and test-retest reliability (Goldberg \& Freyd, 2006). The authors were interested in first-hand trauma experiences (i.e., not witnessing) and so used 7 of the 12 items in data analyses. Two items were classified as low-betrayal, two as medium, and three as high-betrayal. This version of the BBTS differed from the version used in the Kaehler and Freyd (2009) study in two ways: the age categories of the events and the response choices. In the previous study, there were three age categories ("before age 12", "age 12 
through 17", and "age 18 and older") for each event, while for this study there were only two age categories ("before age 18" and "age 18 or older"). Respondents simply selected whether the event occurred ("yes") or did not ("no") in the Kaehler and Freyd (2009) study; in this study, participants selected the frequency it occurred from three response choices ("never", "one or two times", and "more than that"). Scores could range from 0 to 12 for highbetrayal and 0 to 8 for low- and medium-betrayal.

The BPI is a 53-item self-report measure assessing characteristics typical of those diagnosed with BPD. Results may be interpreted with either a dimensional or categorical approach. Sample items include: "my feelings toward other people quickly change into opposite extremes (e.g., from love and admiration to hate and disappointment); if a relationship gets close, I feel trapped; and I enjoy having control over someone"). The BPI has good internal consistency and test-retest reliability (Leichsenring, 1999). The possible responses were revised from the original scale; instead of marking a statement as true/false, participants were given a 5-point Likert-type scale, where $1=$ very inaccurate, $2=$ moderately inaccurate, $3=$ neither accurate nor inaccurate, $4=$ moderately accurate, and $5=$ very accurate. This revision was made in an effort to increase the variability of participant's scores. Furthermore, only 47 of the original 53 items were administered, omitting items that would require follow-up questions.

\section{Procedure}

As previously mentioned, participants completed both instruments by mail as part of a larger, longitudinal data collection project. Participants are compensated for their time. While completing questionnaires, respondents may decline to answer any item. After completing the survey, each questionnaire was marked with an identification number, which was used during data entry so responses are anonymous when analyzing data. IRB approval was obtained prior to the mailing of the questionnaires as well as for this specific series of data analyses.

\section{Results}

\section{Trauma History}

Fifty-one percent of the sample indicated they experienced at least one type of first-hand trauma and $35.8 \%$ experienced at least one high-betrayal trauma. High-betrayal was significantly corre- lated with medium-betrayal $(r=.282, p<.01)$ and mediumbetrayal with low-betrayal $(r=.201, p<.01)$. Gender was significantly associated with high-betrayal $(r=.263, p<.01)$ and low-betrayal $(r=-.102, p=<.01)$, but not with mediumbetrayal $(r=.029, p=\mathrm{NS})$. Women $(M=1.94, S D=2.56)$ had higher scores for high-betrayal experiences than men $(M=.751$, $S D=1.55)$; however, men had higher values $(M=.787, S D=$ 1.14) for low-betrayal events compared to women $(M=.421$, $S D=.768)$ (see Figure 1).

\section{Borderline Personality Traits}

The mean BPI total score was $80.6(S D=18.8)$, median was 77.0 , with a range of 48 to 162 (possible score range of 47 to 235). There was significant positive skew (skewness $=1.09, S E=$ .089). Thirty of the items had $50 \%$ or more respondents endorsing that item as very inaccurate. Twenty of the items had less than $1 \%$ of participants indicating that the statement was very accurate. The scale had good reliability, Cronbach's alpha $=.892$. Ethnicity $(r=$ $.022, p=\mathrm{NS})$, gender $(r=.065, p=\mathrm{NS})$, employment status $(r=$ $.056, p=\mathrm{NS})$, and marital status $(r=.043, p=\mathrm{NS})$ were not significantly correlated with BPI score. All three levels of betrayal events were significantly correlated with BPI score: low $(r=.177$, $p<.01)$, medium $(r=.241, p<.01)$, and high $(r=.249$, $p<.01)$.

\section{Multiple Regression}

To test the hypotheses of interest, a multiple regression analysis was conducted. The model, which included gender and the three levels of betrayal trauma, did significantly predict a portion of the variance in borderline traits, $F(4,581)=21.2, p<.001$, adjusted $r^{2}=.122$. Results of the regression analysis are shown in Table 1. As predicted, high-betrayal trauma was the largest predictor of borderline personality features, $\beta=.201, t(581)=4.74, p<.001$. Medium-betrayal trauma was also a significant predictor $(\beta=$ $.176, t(581)=4.24, p<.001)$. Contrary to predictions, both gender $(\beta=-.099, t(581)=-2.43, p<.05)$ and low-betrayal $(\beta=.126, t(581)=3.15, p<.01)$ did significantly predict variance in BPI scores.

Separate regression models were conducted for men and women to examine how betrayal trauma may differentially associate with borderline personality characteristics based on gender. For men, the model, including all three levels of betrayal trauma, did sig-

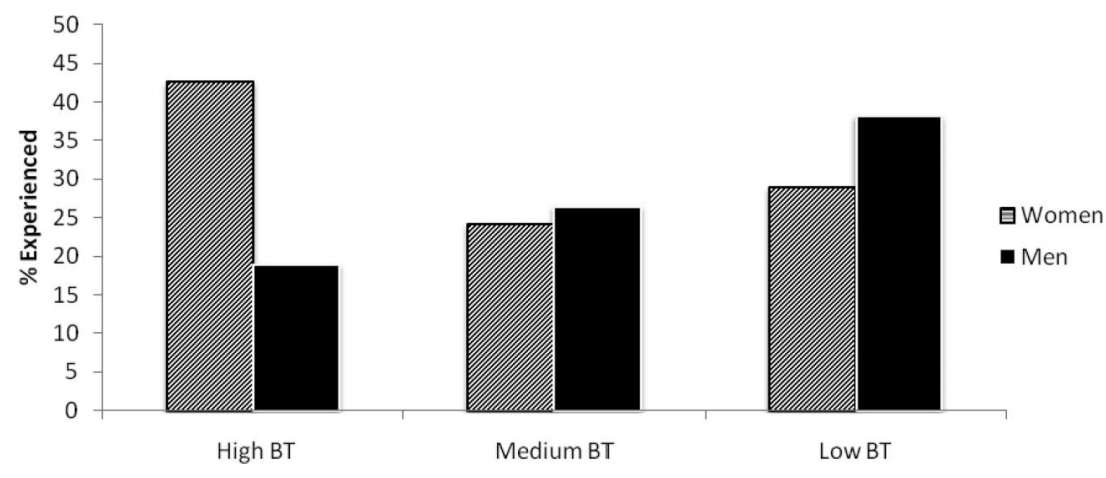

Figure 1. Frequency of betrayal traumas by gender. 
Table 1

Multiple Regression for Betrayal Trauma Predicting BPI $(N=749)$

\begin{tabular}{lccc}
\hline \multicolumn{1}{c}{ Variable } & B & $S E$ B & $\beta$ \\
\hline Gender & 3.71 & 1.53 & $0.99^{*}$ \\
High BT & 1.74 & 0.37 & $0.20^{* * *}$ \\
Medium BT & 4.01 & 0.95 & $0.18^{* * *}$ \\
Low BT & 2.24 & 0.71 & $0.13^{* *}$ \\
\hline
\end{tabular}

Note. $\quad R^{2}=.128$, adjusted $R^{2}=.122$

${ }^{*} p<.05 .{ }^{* *} p<.01 .^{* * * *} p<.001$.

nificantly predict a portion of the variance in borderline traits, $F(3$, $264)=14.4, p<.001$, adjusted $r^{2}=.141$. Results of the regression analysis are shown in Table 2. Medium-betrayal trauma was the largest predictor of borderline personality features, $\beta=$ $.201, t(264)=3.27, p=.001$. Both high-betrayal $(\beta=.154$, $t(264)=2.55, p<.05)$ and low-betrayal $(\beta=.169, t(264)=2.83$, $p<.01$ ) also significantly predicted variance in BPI scores (see Figure 2).

As with men, the model including the three levels of betrayal trauma did significantly predict a portion of the variance in borderline traits, $F(3,314)=13.0, p<.001$, adjusted $r^{2}=.111$. Results of the regression analysis are shown in Table 3 . Highbetrayal trauma was the largest predictor of borderline personality features, $\beta=.229, t(314)=4.02, p<.001$. Medium-betrayal trauma was also a significant predictor $(\beta=.144, t(314)=2.52$, $p<.05)$. However, low-betrayal $(\beta=.081, t(314)=1.50, p=$ .135 ) did not significantly predict variance in BPI scores (see Figure 3).

\section{Discussion}

These findings support an association between betrayal and borderline personality traits. As predicted, high-betrayal traumas were the largest contributor to explained variance of borderline characteristics, and medium-betrayal traumas also significantly predicted borderline features. However, unexpectedly, experiencing traumas with low-betrayal accounted for borderline variance, as did gender. When exploring the relationship between betrayal trauma and borderline personality separately for men and women, interesting results are found. For men, all three types of betrayal traumas predict borderline personality characteristics; however, for women, only experiencing high- and medium-betrayal events predict those characteristics. It was hypothesized that high-and medium-betrayal trauma would be associated with borderline personality characteristics, while low-betrayal would not be, as was

Table 2

Multiple Regression With Betrayal Trauma Predicting BPI for $\operatorname{Men}(N=317)$

\begin{tabular}{lccc}
\hline \multicolumn{1}{c}{ Variable } & B & $S E$ B & $\beta$ \\
\hline High BT & 1.92 & 0.76 & $0.15^{*}$ \\
Medium BT & 4.15 & 1.27 & $0.20^{* * *}$ \\
Low BT & 2.75 & 0.97 & $0.17^{* *}$ \\
\hline
\end{tabular}

${ }^{*} p<.05 . \quad{ }^{* *} p<.01 .{ }^{* * * *} p<.001$.

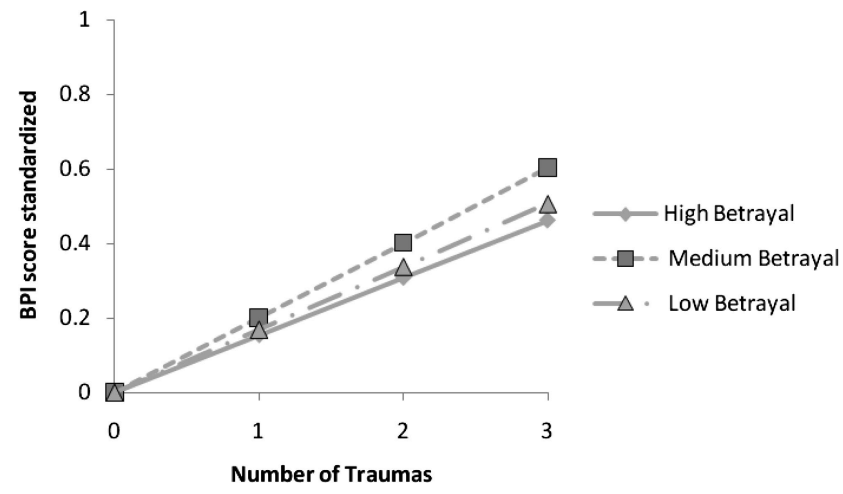

Figure 2. Types of betrayal trauma and standardized male BPI score using standardized $\beta$.

found by Kaehler and Freyd (2009). However, this pattern was only found for women. Thus, for men, the experience of any type trauma results in dysfunctional responses, while for women, only interpersonal traumas do.

DePrince and Freyd (2002) discuss how those in a less powerful position may perceive and respond to betrayal violations more so than those in a more powerful position at the time of the event. Thus, for men, who typically hold positions of power, experiencing any type of trauma may be their first exposure to feelings of disempowerment. A corollary of this is the "just world" construct, the belief the world is "just" and so people are rewarded or punished based on their actions (Janoff-Bulman, 1989; Lerner \& Miller, 1978). Men (O’Connor, Morrison, McLeod, \& Anderson, 1996; Swickert, DeRoma, \& Saylor, 2004), older Americans (Calhoun, Cann, Tedeschi, \& McMillan, 1998), and European Americans (Calhoun, \& Cann, 1994) tend to have higher just world beliefs than women, the younger generation, and African Americans, respectively. Janoff-Bulman (1989), in her work with trauma survivors, found that most people usually operate on the basic belief of invulnerability (including the idea of a just world), which is changed after experiencing a traumatic event. Thus, perhaps for people who have stronger beliefs in a just world (e.g., men), experiencing any type of event that threatens this belief results in maladaptive coping. Recent work by Giesen-Bloo and Arntz (2005) found that BPD patients hold significantly lower beliefs of benevolence of people and benevolence of the world compared to patients with either a Cluster C Personality Disorder, Axis I Disorder, or member of the control group, and that these world assumptions are more attributable to the borderline psychopathology of the person, rather than the experience of trauma. Differ-

Table 3

Multiple Regression With Betrayal Trauma Predicting BPI for Women $(N=432)$

\begin{tabular}{lccc}
\hline \multicolumn{1}{c}{ Variable } & B & SE B & $\beta$ \\
\hline High BT & 1.73 & 0.43 & $0.23^{* *}$ \\
Medium BT & 3.63 & 1.44 & $0.14^{*}$ \\
Low BT & 1.58 & 1.05 & 0.08 \\
\hline
\end{tabular}

$* p<.05$. ** $p<.001$. 


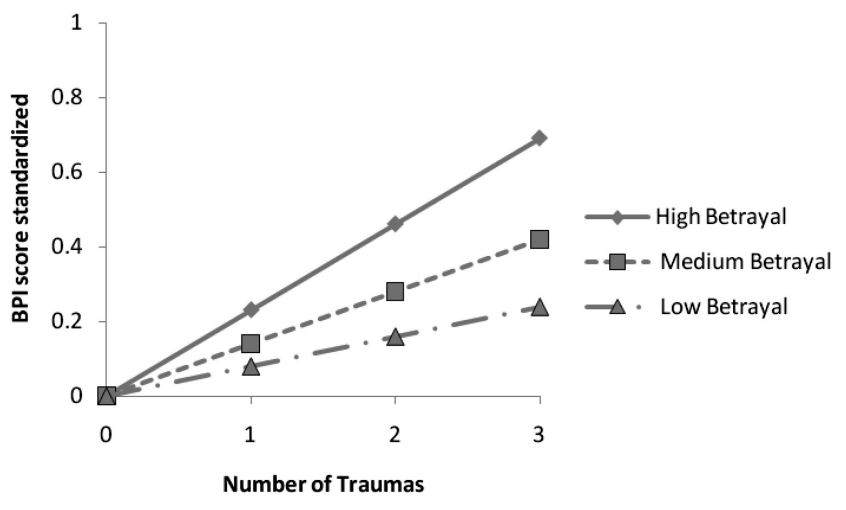

Figure 3. Types of betrayal trauma and standardized female BPI score using standardized $\beta$.

ences in world assumptions may also explain the discrepancy in findings with Kaehler and Freyd (2009), which had a sample primarily composed of women (73\%) compared to this study (57\%). Also, this sample was much older (Mage $=50.7, S D=$ 12.6) compared to the college sample (Mage $=20.1, S D=3.40$ ). Future research should examine the mediating role world assumptions may be playing in the association between betrayal trauma and borderline personality characteristics.

DePrince and Freyd (2002) suggest women may be affected more by interpersonal trauma because women are socialized to attend to interpersonal relationships more so than men, and so will be more impacted by a betrayal of trust in that context compared to a noninterpersonal event. Related, men may focus more on the life-threatening aspects of the trauma rather than the interpersonal; in contrast, women may focus more on the relational dynamics. A study by Cromer and Smyth (2010) supports this with their finding that college women had greater PTSD symptom severity than do college men following interpersonal, but not noninterpersonal, trauma. Future research should be conducted to explore this idea further.

Attachment status may also help explain the observed gender differences. We had initially theorized men might be diagnosed less frequently with BPD because they experience fewer of the high betrayal events, which disrupts attachment (and is characteristic of BPD). Thus, we hypothesized that the event, not gender per se, might explain the gender differences observed in BPD. In support of this approach, Tang and Freyd (in press) found some support for a parallel hypothesis regarding high betrayal exposure and gender differences in PTSD symptoms. However, differences in attachment disruption in response to betrayal events offer an alternative explanation for gender differences in BPD rates. In support of this viewpoint, men have been shown to have higher levels of attachment avoidance and less attachment anxiety compared to women (e.g., Brassard, Shaver, \& Lussier, 2007; Brennan, Clark, \& Shaver, 1998). Attachment anxiety has been demonstrated to correlate with BPD traits but an association between BPD and attachment avoidance is less clear (Scott, Levy, \& Pincus, 2009). Thus, the association between low-betrayal trauma and borderline personality characteristics may be attributable to gender differences in insecure attachment style. Belford, Kaehler, and Birrell (submitted) looked at relational health, which could be considered an attachment proxy, and found that relational health partially mediated betrayal trauma and borderline personality characteristics. However, gender was not significantly correlated with either of those two variables and so was not included in the model. Given its theoretical importance, attachment should be studied further as a potential contributor to the link between betrayal trauma and borderline personality characteristics.

Future research should further explore the connections among trauma, dissociation, and BPD. Although a connection between dissociation and trauma is generally found with BPD, there are reports that do not find this link. For instance, Zweig-Frank and colleagues (1994) did not find an association between childhood trauma and dissociation in those diagnosed with BPD. A later study using a sample of male patients and prisoners (Timmerman \& Emmelkamp, 2001) also suggested no relationship between childhood trauma and dissociation in persons with BPD. However, other studies link specific types of maltreatment to dissociation. Results from Simeon, Nelson, Elias, Greenberg, and Hollander (2003) showed total childhood trauma was not significantly associated with dissociation, but emotional neglect was. Furthermore, one study identified four risk factors for dissociation in BPD patients: "inconsistent treatment by a caretaker, sexual abuse by a caretaker, witnessing sexual violence as a child, and adult rape history" (Zanarini, Ruser, Frankenburg, Hennen, \& Gunderson, 2000, p. 26). While not finding an association between dissociation and sexual abuse, Watson, Chilton, Fairchild, and Whewell (2006) did show dissociation was correlated with physical abuse and emotional neglect, with emotional abuse as the strongest predictor of dissociation. The authors of this study suggest "rather than being an intrinsic component of BPD, dissociation and BPD may share childhood trauma as an etiological factor" (p. 480). As previously stated, BTT would propose the dissociation seen in BPD is acting as a defensive mechanism against the betrayal inherent in childhood trauma in order to prevent threatening information from entering consciousness. The specific link between dissociation and betrayal in predicting BPD should be explored in the future, as well as possible gender differences in this association.

There are several limitations to this study. One limitation of the study is the sample was predominately Caucasian. Although a recent study by Pérez Benítez et al. (2010) showed no racial differences in terms of the association between trauma and BPD, as stated previously, racial differences in beliefs of a just world have been found and therefore could limit generalizability of these results. This limitation can be addressed in future research by oversampling minorities or using a different population. Additionally, the frequency of endorsement of borderline traits was low. Future research should examine how betrayal relates to borderline characteristics in a clinical sample to address this limitation. Furthermore, this study relied solely on self-report methods at one time period and so it is not possible to determine causality. A longitudinal study of borderline characteristics and betrayal trauma would be necessary to determine directionality. Alternatively, as this is a nonexperimental design, the observed results could be attributable to variables not evaluated in this study. Future research can include other variables known to be related to BPD and betrayal traumas, such as dissociation and age of exposure. Despite these limitations, findings from this study support the usefulness of 
betrayal trauma theory for understanding borderline personality disorder.

\section{References}

Agrawal, H. R., Gunderson, J., Holmes, B. M., \& Lyons-Ruth, K. (2004). Attachment studies with borderline patients: A review. Harvard Review of Psychiatry, 12, 94-104. doi:10.1080/10673220490447218

American Psychiatric Association. (2000). Diagnostic and statistical manual of mental disorders (4th ed., text revision). Washington, DC: Author.

Baer, J. C., \& Martinez, C. D. (2006). Child maltreatment and insecure attachment: A meta-analysis. Journal of Reproductive and Infant Psychology, 24, 187-197. doi:10.1080/02646830600821231

Battle, C. L., Shea, M., Johnson, D. M., Yen, S., Zlotnick, C., Zanarini, M. C., . . Morey, L. C. (2004). Childhood maltreatment associated with adult personality disorders: Findings from the collaborative longitudinal personality disorders study. Journal of Personality Disorders, 18, 193211. doi:10.1521/pedi.18.2.193.32777

Belford, B., Kaehler, L. A., \& Birrell, P. (submitted). Relational health as a mediator between betrayal trauma and borderline personality Disorder. Journal of Trauma and Dissociation.

Bernstein, E. M., \& Putnam, F. W. (1996). Development, reliability, and validity of a dissociation scale. Journal of Nervous \& Mental Disease, 174, 727-735. doi:10.1097/00005053-198612000-00004

Bowen, K. (2000). Child abuse and domestic violence in families of children seen for suspected sexual abuse. Clinical Pediatrics, 39, 33-40. doi:10.1177/000992280003900104

Bowlby, J. (1988). A secure base: Parent-child attachment and healthy human development. New York: Basic Books.

Brassard, A., Shaver, P. R., \& Lussier, Y. (2007). Attachment, sexual experience, and sexual pleasure in romantic relationships: A dyadic approach. Personal Relationships, 14, 475-493. doi:10.1111/j.14756811.2007.00166.x

Brennan, K. A., Clark, C. L., \& Shaver, P. R. (1998). Self-report measurement of adult attachment: An integrative overview. In J. A. Simpson \& W. S. Rholes (Eds.), Attachment theory and close relationships (pp. 46-76). New York: Guilford Press.

Calhoun, L., \& Cann, A. (1994). Differences in assumptions about a just world: Ethnicity and point of view. Journal of Social Psychology, 134, 765-770. doi:10.1080/00224545.1994.9923011

Calhoun, L., Cann, A., Tedeschi, R., \& McMillan, J. (1998). Traumatic events and generational differences in assumptions about a just world. Journal of Social Psychology, 138, 789-791. doi:10.1080/ 00224549809603265

Carlson, E. A., Egeland, B., \& Sroufe, L. A. (2009). A prospective investigation of the development of borderline personality symptoms. Development and Psychopathology, 21, 1311-1334. doi:10.1017/ S0954579409990174

Cromer, L. D., \& Smyth, J. M. (2010). Making meaning of trauma: Trauma exposure doesn't tell the whole story. Journal of Contemporary Psychotherapy, 40, 65-72. doi:10.1007/s10879-009-9130-8

DePrince, A. P., \& Freyd, J. J. (2001). Memory and dissociative tendencies: The roles of attentional context and word meaning in a directed forgetting task. Journal of Trauma \& Dissociation, 2, 67-82. doi: 10.1300/J229v02n02_06

DePrince, A. P., \& Freyd, J. J. (2002). The intersection of gender and betrayal. In R. Kimerling, P. Ouimette, \& J. Wolfe (Eds.), Gender and PTSD (pp. 98-113). New York: Guilford Press.

Freyd, J. J. (1996). Betrayal trauma: The logic of forgetting childhood abuse. Cambridge, MA: Harvard University Press.

Giesen-Bloo, J., \& Arntz, A. (2005). World assumptions and the role of trauma in borderline personality disorder. Journal of Behavior Therapy and Experimental Psychiatry, 36, 197-208.

Goldberg, L. R. (1999a). A broad-bandwidth, public-domain, personality inventory measuring the lower-level facets of several five-factor models. In I. Mervielde, I. Deary, F. De Fruyt, \& F. Ostendorf (Eds.), Personality psychology in Europe, Vol. 7 (pp. 7-28). Tilburg, The Netherlands: Tilburg University Press.

Goldberg, L. R. (1999b). The Curious Experiences Survey, a revised version of the Dissociative Experiences Scale: Factor structure, reliability, and relations to demographic and personality variables. Psychological Assessment, 11, 134-145. doi:10.1037/1040-3590.11.2.134

Goldberg, L. R., \& Freyd, J. J. (2006). Self-reports of potentially traumatic experiences in an adult community sample: Gender differences and test-retest stabilities of the items in a brief betrayal-trauma survey. Journal of Trauma \& Dissociation, 7, 39-63. doi:10.1300/ J229v07n03_04

Gunderson, J. G., \& Lyons-Ruth, K. (2008). BPD's interpersonal hypersensitivity phenotype: Gene-environment-developmental model. Journal of Personality Disorders, 22, 22-41. doi:10.1521/pedi.2008.22.1.22

Herman, J. L., Perry, J., \& van der Kolk, B. A. (1989). Childhood trauma in borderline personality disorder. American Journal of Psychiatry, 146, 490-495.

Janoff-Bulman, R. (1989). Assumptive worlds and the stress of traumatic events: Applications of the schema construct. Social Cognition, 7, 113136. doi:10.1521/soco.1989.7.2.113

Johnson, D. M., Shea, M. T., Yen, S., Battle, C. L., Zlotnick, C., Sanislow, C. A., ... Zanarini, M. C. (2003). Gender differences in borderline personality disorder: Findings from the collaborative longitudinal personality disorders study. Comprehensive Psychiatry, 44, 284-292. doi: 10.1016/S0010-440X(03)00090-7

Johnson, J. J., Smailes, E. M., Cohen, P., Brown, J., \& Bernstein, D. P. (2000). Associations between four types of childhood neglect and personality disorder symptoms during adolescence and early adulthood: Findings of a community-based longitudinal study. Journal of Personality Disorders, 14, 171-187. doi:10.1521/pedi.2000.14.2.171

Kaehler, L. A., \& Freyd, J. J. (2009). Borderline personality characteristics: A betrayal trauma approach. Psychological Trauma: Theory, Research, Practice, and Policy, 1, 261-268. doi:10.1037/a0017833

Lamb, M. E., Gaensbauer, T. J., Malkin, C. M., \& Schultz, L. A. (1985). The effects of child maltreatment on security of infant-adult attachment. Infant Behavior \& Development, 8, 35-45. doi:10.1016/S01636383(85)80015-1

Leichsenring, F. F. (1999). Development and first results of the Borderline Personality Inventory: A self-report instrument for assessing borderline personality organization. Journal of Personality Assessment, 73, 45-63. doi:10.1207/S15327752JPA730104

Lerner, M. J., \& Miller, D. T. (1978). Just world research and the attribution process: Looking back and ahead. Psychological Bulletin, 85, 1030-1051. doi:10.1037/0033-2909.85.5.1030

Levy, K. N. (2005). The implications of attachment theory and research for understanding borderline personality disorder. Development and Psychopathology, 17, 959-986. doi:10.1017/S0954579405050455

Lyons-Ruth, K., Yellin, C., Melnick, S., \& Atwood, G. (2005). Expanding the concept of unresolved mental states: Hostile/helpless states of mind on the Adult Attachment Interview are associated with disrupted mother-infant communication and infant disorganization. Development and Psychopathology, 17, 1-23. doi:10.1017/S0954579405050017

Minzenberg, M. J., Poole, J. H., \& Vinagradov, S. (2006). Adult social attachment disturbance is related to childhood maltreatment and current symptoms in borderline personality disorder. The Journal of Nervous and Mental Disease, 194, 341-348. doi:10.1097/01.nmd.0000218341.54333.4e

O'Connor, W. E., Morrison, T. G., McLeod, L. D., \& Anderson, D. (1996). A meta- analytic review of the relationship between gender and belief in a just world. Journal of Social Behavior and Personality, 11, 141-148.

Paris, J. (1997). Childhood trauma as an etiological factor in the personality disorders. Journal of Personality Disorders, 11, 34-49. doi:10.1521/ pedi.1997.11.1.34 
Pérez Benítez, C. I., Yen, S., Shea, M. T., Edelen, M. O., Markowitz, J. C., McGlashan, T. H., ... Morey, L. C. (2010). Ethnicity in trauma and psychiatric disorders: Findings from the collaborative longitudinal study of personality disorders. Journal of Clinical Psychology, 66, 583-598.

Rogosch, F. A., \& Cicchetti, D. (2005). Child maltreatment, attention networks, and potential precursors to borderline personality disorder. Development and Psychopathology, 17, 1071-1089. doi:10.1017/ S0954579405050509

Ross, C. A. (2007). Borderline personality disorder and dissociation. Journal of Trauma and Dissociation, 8, 71-80. doi:10.1300/J229v08n01_05

Scott, L. N., Levy, K. N., \& Pincus, A. L. (2009). Adult attachment, personality traits, and borderline personality disorder features in young adults. Journal of Personality Disorders, 23, 258-280. doi:10.1521/ pedi.2009.23.3.258

Simeon, D., Nelson, D., Elias, R., Greenberg, J., \& Hollander, E. (2003). Relationship of personality to dissociation and childhood trauma in borderline personality disorder. CNS Spectrums, 8, 760-762.

Skodol, A. E., Gunderson, J. G., Pfohl, B., Widiger, T. A., Livesley, W., \& Siever, L. J. (2002). The borderline diagnosis I: Psychopathology, comorbidity, and personality structure. Biological Psychiatry, 51, 936950. doi:10.1016/S0006-3223(02)01324-0

Swickert, R., DeRoma, V. M., \& Saylor, C. F. (2004). The relationship between gender and trauma symptoms: A proposed mediational model. Individual Differences Research, 2, 203-223.

Tang, S. S., \& Freyd, J. J. (in press). Betrayal trauma and gender differences in posttraumatic stress. Psychological Trauma: Theory, Research, Practice, and Policy.

Timmerman, I. G., \& Emmelkamp, P. M. (2001). The relationship between traumatic experiences, dissociation, and borderline personality pathology among male forensic patients and prisoners. Journal of Personality Disorders, 15, 136-149. doi:10.1521/pedi.15.2.136.19215

Trull, T. J. (2001). Structural relations between borderline personality disorder features and putative etiological correlates. Journal of Abnormal Psychology, 110, 471-481. doi:10.1037/0021-843X.110.3.471

Watson, S., Chilton, R., Fairchild, H., \& Whewell, P. (2006). Association between childhood trauma and dissociation among patients with borderline personality disorder. Australian and New Zealand Journal of Psychiatry, 40, 478-481.

Wildgoose, A., Waller, G., Clarke, S., \& Reid, A. (2000). Psychiatric symptomatology in borderline and other personality disorders: Dissociation and fragmentation as mediators. Journal of Nervous and Mental Disease, 188, 757-763. doi:10.1097/00005053-200011000-00006

Woodward, H. E., Taft, C. T., Gordon, R. A., \& Meis, L. A. (2009). Clinician bias in the diagnosis of posttraumatic stress disorder and borderline personality disorder. Psychological Trauma: Theory, Research, Practice, and Policy, 1, 282-290. doi:10.1037/a0017944

Zanarini, M. C. (2000). Childhood experiences associated with the development of borderline personality disorder. Psychiatric Clinics of North America, 23, 89-101. doi:10.1016/S0193-953X(05)70145-3

Zanarini, M. C., Ruser, T. F., Frankenburg, F. R., Hennen, J., \& Gunderson, J. G. (2000). Risk factors associated with the dissociative experiences of borderline patients. Journal of Nervous \& Mental Disease, 188, 26-30. doi:10.1097/00005053-200001000-00005

Zanarini, M. C., Williams, A. A., Lewis, R. E., Reich, R. B., Vera, S. C., Marino, M. F., ... Frankenburg, F. R. (1997). Reported pathological childhood experiences associated with the development of borderline personality disorder. The American journal of psychiatry, 154, 11011106.

Zweig-Frank, H., Paris, J., \& Guzder, J. (1994). Psychological risk factors for dissociation and self-mutilation in female patients with borderline personality disorder. Canadian Journal of Psychiatry, 39, 259-264.

Received October 10, 2010

Revision received April 8, 2011

Accepted April 19, 2011 